PROCEEDINGS OF THE

AMERICAN MATHEMATICAL SOCIETY

Volume 140, Number 1, January 2012, Pages 161-165

S 0002-9939(2011)10914-6

Article electronically published on May 2, 2011

\title{
ON HARMONIC FUNCTIONS AND THE SCHWARZ LEMMA
}

\author{
DAVID KALAJ AND MATTI VUORINEN
}

(Communicated by Richard Rochberg)

\begin{abstract}
We study the Schwarz lemma for harmonic functions and prove sharp versions for the cases of real harmonic functions and the norm of harmonic mappings.
\end{abstract}

\section{INTRODUCTION}

We first recall that the hyperbolic metric $d_{h}(z, w)$ of the unit disk $\mathbf{U}:=\{z$ : $|z|<1\}$ is defined by

$$
\tanh \frac{d_{h}(z, w)}{2}=\frac{|z-w|}{|1-z \bar{w}|} .
$$

The classical Schwarz lemma says that an analytic function $f$ of the unit disk into itself is a contraction in the hyperbolic metric, i.e. for $z, w \in \mathbf{U}$,

$$
\frac{|f(z)-f(w)|}{|1-f(z) \overline{f(w)}|} \leqslant \frac{|z-w|}{|1-z \bar{w}|} \text {. }
$$

Letting $z \rightarrow w$ we get

$$
\left|f^{\prime}(z)\right| \leqslant \frac{1-|f(z)|^{2}}{1-|z|^{2}}
$$

In the particular case $f(0)=0$ we obtain from (1.1) the following:

Lemma 1.3. Let $f: \mathbf{U} \rightarrow \mathbf{U}$ be an analytic function with $f(0)=0$. Then $|f(z)| \leqslant$ $|z|, z \in \mathbf{U}$.

In this paper we will consider harmonic functions $f: \mathbf{U} \rightarrow B_{n}:=\{x:|x|<$ 1\} $\subset \mathbf{R}^{n}$, i.e. functions whose coordinate functions satisfy the Laplace equation. In this case, the Cauchy-Riemann equations need not be satisfied. During the past decade, harmonic functions have been extensively studied, and many results from the theory of analytic functions have been extended for them. For instance, the Schwarz lemma has the following counterpart in this context.

Lemma 1.4 ([3], [2, p. 77] and [7, Theorem 4.4.6]). Let $f: \mathbf{U} \rightarrow \mathbf{U}$ be harmonic functions with $f(0)=0$. Then $|f(z)| \leqslant \frac{4}{\pi} \tan ^{-1}|z|$, and this inequality is sharp for each point $z \in \mathbf{U}$.

Recently, several authors have proved the following three extensions of the Schwarz lemma, which is the motivation for our study.

Received by the editors October 23, 2010.

2010 Mathematics Subject Classification. Primary 31C05; Secondary 30A10.

Key words and phrases. Harmonic functions, hyperbolic metric, unit disk.

(C)2011 American Mathematical Society 
Proposition 1.5 ([1, Theorem 6.26]). Let $f$ be a real harmonic function of the unit disk into $(-1,1)$. Then the following sharp inequality holds:

$$
|\nabla f(0)| \leqslant \frac{4}{\pi}
$$

Proposition 1.6 ([5, Proposition 3.1]). Let $f$ be a K-q.c. harmonic mapping of the unit disk into itself. Then

$$
|\nabla f(z)| \leqslant K \frac{1-|f(z)|^{2}}{1-|z|^{2}} .
$$

The previous proposition has its counterpart for the class of quasiconformal hyperbolic harmonic mappings. See [8] for related results.

Proposition 1.7 ([6, Theorem 1.1]). Let $f$ be an analytic function of the unit disk into the unit ball $B_{n} \subset \mathbf{C}^{n}$. Then the following sharp inequality holds:

$$
|\nabla| f(z)|| \leqslant \frac{1-|f(z)|^{2}}{1-|z|^{2}} .
$$

The results of this paper are:

Theorem 1.8. Let $f$ be a real harmonic function of the unit disk into $(-1,1)$. Then the following sharp inequality holds:

$$
|\nabla f(z)| \leqslant \frac{4}{\pi} \frac{1-|f(z)|^{2}}{1-|z|^{2}}, \quad|z|<1 .
$$

Theorem 1.10. If $f$ is a harmonic mapping of the unit disk into the unit ball $B_{n} \subset \mathbf{R}^{n}$, then

$$
|\nabla| f(z)|| \leqslant \frac{4}{\pi} \frac{1-|f(z)|^{2}}{1-|z|^{2}}, \quad|z|<1 .
$$

The inequality (1.11) is sharp for each $z$.

Theorem 1.12. Let $f$ be a harmonic function of the unit disk into $(-1,1)$. Then we have

$$
d_{h}(f(z), f(w)) \leqslant \frac{4}{\pi} d_{h}(z, w),
$$

where $d_{h}$ on the left (right) side is the hyperbolic distance of $(-1 ; 1)$ (of $U$ ), respectively.

Remark 1.13. Theorem 1.8 is an improvement and an extension of Proposition 1.5 Notice that

$$
\frac{4}{\pi}\left(1-|f(0)|^{2}\right) \leqslant \frac{4}{\pi} .
$$

Theorem 1.10 is a harmonic version of Proposition 1.7.

The following example is taken from [4, but it is used there in some other context. It shows that we do not have any complex version of Theorem 1.8, without imposing quasiconformality (as is done in Proposition 1.6).

Example 1.14. Let $z=r e^{i t}$ and let $f$ be a harmonic diffeomorphism of the unit disk onto itself such that $\partial_{r} f$ exists in the boundary point 1 and $\partial_{t} f$ is not bounded on $z=1$. Then

$$
\varlimsup_{r \rightarrow 1} \frac{1-|f(r)|^{2}}{1-r^{2}}=\left.|f(1)| \frac{\partial f(r)}{d r}\right|_{r=1}=M<\infty,
$$


but

$$
\sup _{r \in[0,1)}|\nabla f(r)|=\infty .
$$

For instance, let $\psi$ be the function defined on $[-\pi, \pi]$ by

$$
\psi(\theta)=a\left(\theta+\int_{0}^{\theta}-\log |x| d x\right),
$$

where $a$ is a positive constant such that

$$
a\left(2 \pi+\int_{-\pi}^{\pi}-\log |x| d x\right)=2 \pi .
$$

Take $F(\theta)=e^{i \psi(\theta)}$ and define $f(z)=P[F](z)$.

\section{Proofs}

Proof of Theorem 1.8. Let $h$ be the harmonic conjugate of $f$. Then $a=f+i h$ maps the unit disk $\mathbf{U}$ into the vertical strip $S=\{w:-1<\Re w<1\}$.

Making use of the conformal map

$$
g(z)=\frac{2 i}{\pi} \log \frac{1+z}{1-z}
$$

of the unit disk $\mathbf{U}$ onto the strip $S$, we see that there exists an analytic function $b: \mathbf{U} \rightarrow \mathbf{U}$ such that

$$
a(z)=\frac{2 i}{\pi} \log \frac{1+b(z)}{1-b(z)} .
$$

By the Schwarz lemma for analytic functions we have

$$
\left|b^{\prime}(z)\right| \leqslant \frac{1-|b(z)|^{2}}{1-|z|^{2}} .
$$

On the other hand,

$$
a^{\prime}(z)=\frac{4 i}{\pi} \frac{b^{\prime}(z)}{1-b(z)^{2}}
$$

and

$$
|\nabla f|=\left|a^{\prime}(z)\right| \text {. }
$$

We will find the best possible constant $C$ such that

$$
|\nabla f(z)| \leqslant C \frac{1-|f(z)|^{2}}{1-|z|^{2}} .
$$

As

$$
\left|a^{\prime}(z)\right| \leqslant \frac{4}{\pi} \frac{1-|b(z)|^{2}}{\left|1-b(z)^{2}\right|} \frac{1}{1-|z|^{2}},
$$

it will be enough to find the best possible constant $C$ such that

$$
\frac{4}{\pi} \frac{1-|b(z)|^{2}}{\left|1-b(z)^{2}\right|} \frac{1}{1-|z|^{2}} \leqslant C \frac{1-|\Re a(z)|^{2}}{1-|z|^{2}}
$$

or, what is the same,

$$
\frac{4 \pi}{\left(\pi^{2}-4\left|\arg \frac{1+b}{1-b}\right|^{2}\right)} \frac{1-|b|^{2}}{\left|1-b^{2}\right|} \leqslant C,|b|<1 .
$$


Let $\omega=\frac{1+b}{1-b}=r e^{i t}$. Then $-\pi / 2 \leqslant t \leqslant \pi / 2$ and

$$
\begin{aligned}
& 1-|b|^{2}=\frac{4 r \cos t}{r^{2}+2 r \cos t+1}, \\
& \left|1-b^{2}\right|=\frac{4 r\left|e^{i t}\right|}{r^{2}+2 r \cos t+1},
\end{aligned}
$$

and hence the last inequality with the constant $C=4 / \pi$ follows from

$$
\frac{|\cos t|}{1-\frac{4}{\pi^{2}} t^{2}} \leqslant 1
$$

which holds for $t \in(-\pi / 2, \pi / 2)$. This yields (1.9).

To show that the inequality (1.9) is sharp, take the harmonic function

$$
f(z)=\frac{2}{\pi} \arctan \frac{2 y}{1-x^{2}-y^{2}} .
$$

It is easy to see that

$$
|\nabla f(0)|=\frac{4}{\pi}=\frac{4}{\pi} \frac{1-|f(0)|^{2}}{1-0^{2}} .
$$

Proof of Theorem 1.10. Consider the function $g(z)=\langle f(z), b\rangle$, where $|b|=1, b \in$ $\mathbf{R}^{n}$. Then by Theorem 1.8, for $|h|=1, h \in \mathbf{C}$, we have

$$
\langle\nabla f(z) h, b\rangle \leqslant \frac{4}{\pi} \frac{1-|g(z)|^{2}}{1-|z|^{2}} .
$$

Let

$$
b=\frac{f(z)}{|f(z)|}
$$

and

$$
h=\frac{\nabla f(z)^{t} b}{\left|\nabla f(z)^{t} b\right|} .
$$

Here $\nabla f(z)^{t}$ is the transpose of the matrix $\nabla f(z)$. Then (2.1) coincides with

$$
|\nabla| f|| \leqslant \frac{4}{\pi} \frac{1-|f(z)|^{2}}{1-|z|^{2}} .
$$

The desired conclusion follows.

Proof of Theorem 1.12. Let $m$ be a Möbius transformation of the unit disk onto itself that maps the points 0 and $r>0$ to the points $z$ and $w$. Then the function $f \circ m$ is also harmonic. Denote it by $f$ as well. Without loss of generality assume that $f(z) \leqslant f(w)$. Let $C=\left\{t \in[0, r]: f^{\prime}(t)>0\right\}$. Then $C=\bigcup_{n=0}^{\infty} C_{n}$, where $C_{n}$ are some intervals in $[0, r]$. Let $A_{1}=C_{1}, A_{2}=C_{2} \backslash\left\{t: f(t) \in f\left(A_{1}\right)\right\}$, and by induction, let

$$
A_{n+1}=\left\{t \in C_{n}: f(t) \notin f\left(\bigcup_{k=1}^{n} A_{k}\right)\right\} .
$$

Take

$$
A=\bigcup_{k=1}^{\infty} A_{k}
$$


Denote by $|\cdot|$ the Lebesgue measure on the real line. Then $[f(z), f(w)] \subset f([0, r])$ and $A$ is a measurable subset of $[0, r]$ such that $|[f(z), f(w)]|=|f(A)|$ and $f$ is injective in $A$. Therefore by using (1.9) we have

$$
\begin{aligned}
d_{h}(f(z), f(w)) & =\int_{f(z)}^{f(w)} \frac{|d \omega|}{1-|\omega|^{2}} \\
& =\int_{A} \frac{|d f(t)|}{1-|f(t)|^{2}} \\
& \leqslant \frac{4}{\pi} \int_{A} \frac{|d t|}{1-|t|^{2}} \\
& \leqslant \frac{4}{\pi} \int_{[0, r]} \frac{|d t|}{1-|t|^{2}}=\frac{4}{\pi} d_{h}(z, w) .
\end{aligned}
$$

\section{ACKNOWLEDGMENTS}

This work was completed during the visit of the first author to the Universities of Helsinki and Turku in October 2010. The first author was partially supported by the Ministry of Education of Montenegro. This research was supported by the Academy of Finland, project 2600066611.

\section{REFERENCES}

[1] S. Axler, P. Bourdon, W. Ramey, Harmonic function theory, Springer Verlag, New York, 2000. MR.1184139 (93f:31001)

[2] P. Duren, Harmonic mappings in the plane, Cambridge University Press, 2004. MR2048384 (2005d:31001)

[3] E. Heinz, On one-to-one harmonic mappings, Pacific J. Math. 9 (1959), 101-105. MR0104933 $(21: 3683)$

[4] D. Kalaj, Harmonic functions and harmonic quasiconformal mappings between convex domains, thesis, Beograd, 2002.

[5] M. Knežević, M. Mateljević, On the quasi-isometries of harmonic quasiconformal mappings, J. Math. Anal. Appl. 334 (1) (2007), 404-413. MR2332565 (2008e:30025)

[6] M. Pavlović, A Schwarz lemma for the modulus of a vector-valued analytic function, Proc. Amer. Math. Soc. 139 (2011), 969-973.

[7] M. Pavlović, Introduction to function spaces on the disk. Posebna Izdanja, 20. Matematički Institut SANU, Belgrade, 2004. MR2109650 (2006d:30001)

[8] T. Wan, Constant mean curvature surface, harmonic maps, and universal Teichmüller space, J. Differential Geom. 35 (3) (1992), 643-657. MR.1163452 (94a:58053)

Faculty of Natural Sciences and Mathematics, University of Montenegro, Cetinjski PUt B.B., 81000 Podgorica, Montenegro

E-mail address: davidk@t-com.me

Department of Mathematics, University of Turku, 20014 Turku, Finland

E-mail address: vuorinen@utu.fi 